\title{
Genetic Instability in Patients with Hodgkin's Disease Undergoing Chemotherapy
}

\author{
Jorge M. Abdallah, Donald P. Lombardi, and Ilan R. Kirsch \\ National Cancer Institute-Navy Medical Oncology Branch, Bethesda, Maryland 20889-5105
}

\begin{abstract}
We have studied the effect of chemotherapy on the level of a particular kind of genetic instability in patients with Hodgkin's disease. The particular type of genetic instability assayed is exemplified by trans-rearrangements between two ( rather than within one) $\mathrm{T}$ cell antigen receptor. 16 patients were studied during their course of treatment. Presentation samples were available for 13 of these patients; 9 of them showed an increase in the level of trans-rearrangements during their exposure to chemotherapeutic agents $(P<0.043)$. All patients for whom posttherapy samples were available (10 out of 16) showed a return to baseline levels of transrearrangements 1-5 mo after completion of therapy $(P$ $<0.03$ ). Thus, this assay appears to be a marker for the "destabilizing" effects of certain chemotherapeutic agents. (J. Clin. Invest. 1995. 96:2744-2747.) Key words: T cell • gene rearrangement $\bullet$ malignancy
\end{abstract}

\section{Introduction}

Genetic instability is a fact of life. It is the basis of evolution and also the basis of cancer. An individual's risk of developing a particular kind of malignancy is a reflection of that person's propensity to develop genetic mutations, deletions, amplifications, insertions, or translocations, in general, or in specific lineages $(1,2)$. We have developed a PCR-based assay that measures a certain kind of instability specific to lymphocytes. It is defined by the frequency of occurrence of variable (V), ${ }^{1}$ diversity (D), joining $(\mathrm{J})$ recombinase-mediated trans-rearrangements between a $\mathrm{V}$ segment from the $\mathrm{T}$ cell receptor gamma (7p14-15) locus and a $J$ segment from the $T$ cell receptor beta $(7 \mathrm{q} 35)$ locus $(3)$. This rearrangement results in the inversion of chromosome 7. This karyotypic abnormality is found at a low frequency in all individuals. Increases in these trans-rearrangements are seen in patients with the autosomal

Address correspondence to Ilan R. Kirsch, Section Chief National Cancer Institute-Navy Medical Oncology Branch, Bldg. 8, Room 5101, National Naval Medical Center, 8901 Rockville Pike, Bethesda, MD 20889-5105. Phone: 301-496-0909; Fax: 301-496-0047.

Received for publication 5 June 1995 and accepted in revised form 7 September 1995

1. Abbreviations used in this paper: $\mathrm{ABV}$, adriamycin, bleomycin, and vinblastine; $\mathrm{ABVD}, \mathrm{ABV}$ and decarbazine; $\mathrm{AT}$, ataxia-telangiectasia; $\mathrm{EPOCH}$, etoposide, vincristine, doxorubicin, cyclophosphamide, and prednisone; J, joining; MOPP, mechlorethamine, vincristine, procarbazine, and prednisone; $\mathrm{V}$, variable.

The Journal of Clinical Investigation, Inc.

Volume 96, December 1995, 2744-2747 recessive syndrome ataxia-telangiectasia (AT). AT is characterized by progressive cerebellar degeneration, oculocutaneous telangectasia, premature aging, immunodeficiency, radiosensitivity, chromosomal breakage, and an increased risk of developing lymphoid malignancy. The frequency of interlocus recombination is $10-100$-fold higher in AT than in non-AT individuals (4). Coincidentally or not, their increased risk of the development of cancer is also $10-100$-fold higher $(5,6)$. Increased frequency of interlocus recombination of $\mathrm{T}$ cell receptor genes is not only inherited, as in AT, but can be induced. In a group of agricultural workers from the "leukemia-lymphoma" belt of southern Minnesota and northern Iowa (7), it was found that increased frequency of interlocus recombination was correlated with exposure to herbicides, pesticides, and fumigants (including phosphine, malathion, dichlorophenoxy acetic acid, chloropicrin, and captan). The observed increase seemed to be related to the amount of these agents used; seasonal variation in the level of trans-rearrangements was also documented. In this group a 15-30-fold increased frequency of interlocus recombination was observed (compared with matched controls) (7). These agricultural workers also have a three- to sevenfold increased risk of developing a lymphoid malignancy (8).

Patients with Hodgkin's disease treated with radiotherapy, chemotherapy, or both can be cured in almost $80 \%$ of all cases. However, their relative risk of developing secondary malignancy is 34.7 for leukemia and 20.6 for non-Hodgkin's lymphoma $(9,10) .16$ patients treated with chemotherapy for Hodgkin's disease were evaluated for their frequency of $\mathrm{V} \gamma /$ $\mathrm{J} \beta$ trans-rearrangements before, during, and after completion of therapy.

\section{Methods}

16 patients with Hodgkin's disease were included in this study (Table I). They were all treated at the National Cancer Institute, nine at the National Cancer Institute-Navy Medical Oncology Branch and seven at the National Cancer Institute-Medicine Branch. There were nine males and seven females. Their ages ranged from 21 to $55 \mathrm{yr}$ old. At the time of diagnosis, there were six patients with stage IIA, four stage IIB, three stage IIIA, two stage IIIB, and one stage IVA. Nine were newly diagnosed patients, and seven were patients with relapsed disease at the time of entry in our study. Seven patients received adriamycin, bleomycin, vinblastine, and decarbazine (ABVD), six received etoposide, vincristine, doxorubicin, cyclophosphamide, and prednisone (EPOCH), and one received mechlorethamine, vincristine, procarbazine, and prednisone (MOPP). One patient was treated with MOPP/ABVD and one with MOPP/adriamycin, bleomycin, and vinblastine (ABV) hybrid. For these two patients chemotherapy was followed by radiotherapy. Mononuclear cells were obtained by Ficoll gradient centrifugation from heparinized blood on the starting day of each chemotherapy pulse as well as on follow-up visits after completion of therapy. Genomic DNA was isolated by modified method of Buffone procedure (11) and resuspended in deionized water at a concentration of $100 \mathrm{ng} / \mu \mathrm{l}$. DNA concentration was measured by spectrophotometry and rechecked by agarose gel elec- 
Table I. Hodgkin's Disease Patients Clinicopathological Data

\begin{tabular}{rrllccllc}
\hline Patient & Age & Sex & Stage & $\begin{array}{c}\text { First } \\
\text { diagnosis }\end{array}$ & Relapse & Therapy & $\begin{array}{c}\text { Histology } \\
\text { type }\end{array}$ \\
\hline 1 & 45 & M & IIA & & X & EPOCH & NS \\
2 & 24 & M & IIB & & X & EPOCH & NS \\
3 & 36 & F & IIIB & & X & EPOCH & NS \\
4 & 54 & M & IIA & & X & EPOCH & NS \\
5 & 26 & F & IVA & & X & EPOCH & NS \\
6 & 25 & F & IIB & & X & EPOCH & NS \\
7 & 29 & F & III & X & & ABVD & MC \\
8 & 32 & F & IIIA & X & & ABVD & LD \\
9 & 34 & F & IIA & X & & ABVD & NS \\
10 & 26 & M & IIA & X & & ABVD & MC \\
11 & 44 & M & IIIA & X & & ABVD & MC \\
12 & 21 & F & IIB & X & & ABVD & NS \\
13 & 34 & M & IIA & X & & ABVD & MC \\
14 & 22 & M & IIA & X & & MOPP/ABVD & NS \\
15 & 49 & M & IIB & X & & MOPP/ABV & LD \\
16 & 55 & M & IIIA & & X & MOPP & LP
\end{tabular}

NS, nodular sclerosing, MC, mixed cellularity, LP, lymphocyte predominant, LD, lymphocyte depleted.

trophoresis. For PBMCs we routinely extracted $1 \mu \mathrm{g}$ DNA/1.5-2.0 $\times$ $10^{5}$ cells. To assay for recombinations between $\mathrm{V} \gamma$ and $\mathrm{J} \beta$ segments, a nested PCR was performed as previously described $(4,7)$. Briefly, in the first step DNA $(1,000,500,250$, and $125 \mathrm{ng})$ was suspended in a $50-\mu \mathrm{l}$ solution containing $200 \mu \mathrm{M}$ deoxynucleotides $50 \mathrm{mM} \mathrm{KCl}, 10$ $\mathrm{mM}$ Tris, $\mathrm{pH} 8.3,1.5 \mathrm{mM} \mathrm{MgCl}$, and $0.01 \%$ gelatin, the "a" set of primers ( $\mathrm{V} \gamma \mathrm{a}$ and $\mathrm{J} \beta 1 \mathrm{a})$ at concentration of $1.4 \mathrm{ng} / \mu \mathrm{l}, 10 \% \mathrm{DMSO}$, and $2.5 \mathrm{U}$ of Taq polymerase. Negative and positive control samples were run with each experiment. The reaction was carried out at $95^{\circ} \mathrm{C}$ $\times 4$ min for denaturation, followed by 25 cycles of amplification consisting of $95^{\circ} \mathrm{C} \times 15 \mathrm{~s}, 57.5^{\circ} \mathrm{C} \times 15 \mathrm{~s}$, and $72^{\circ} \mathrm{C} \times 30 \mathrm{~s}$ plus $6 \mathrm{~s}$ increase per cycle. After 25 cycles, $10 \mathrm{~min}$ at $72^{\circ} \mathrm{C}$ for elongation was allowed. $10 \%$ of the first step reaction was nested using the same conditions with $a$ " $b$ " set of primers $(\mathrm{V} \gamma \mathrm{b}$ and $\mathrm{J} \beta 1 \mathrm{~b})$ at a concentration of $6 \mathrm{ng} / \mu \mathrm{l}$. PCR products were run in $1.5 \%$ agarose electrophoretic gel, and a picture of ethidium bromide-labeled DNA products was taken before transferring to a nylon paper. A " $c$ " set of primers was used for hybridization of the Southern blots of PCR products to verify $\mathrm{V} \gamma / \mathrm{J} \beta$ hybrids. Primer sequences have been previously published $(4,7)$. A product was called positive for $\mathrm{V} \gamma / \mathrm{J} \beta$ rearrangements if it hybridized to both probes $(\mathrm{V} \gamma \mathrm{c}$ and $\mathrm{J} \beta 1 \mathrm{c})$. Values were expressed as the reciprocal of the dilution titer per microgram of DNA. Results of samples obtained during therapy were compared to results of samples obtained before chemotherapy ( 13 patients) and after chemotherapy (10 patients). "PCR-ability" of DNA was assessed using an actin gene. The average number of samples analyzed per patient was: 1 pretherapy, 6.2 during therapy, and 1.7 after therapy. The average interval after cessation of therapy samples was 4 mo (range 0.5-9).

Statistical analysis. The rearrangement frequencies during therapy were compared to the rearrangement frequencies before and after therapy using a paired $t$ test (12). To reduce the impact of a few very high frequencies (providing a "conservative" interpretation of the data), a square root transformation was applied to the individual rearrangement frequencies for each patient before using paired $t$ test to compare the mean during therapy to the mean before and after therapy.

\section{Results}

Our results show an increased level of $\mathrm{V} \gamma / \mathrm{J} \beta$ trans-rearrangements of the $T$ cell receptor genes in PBL of patients

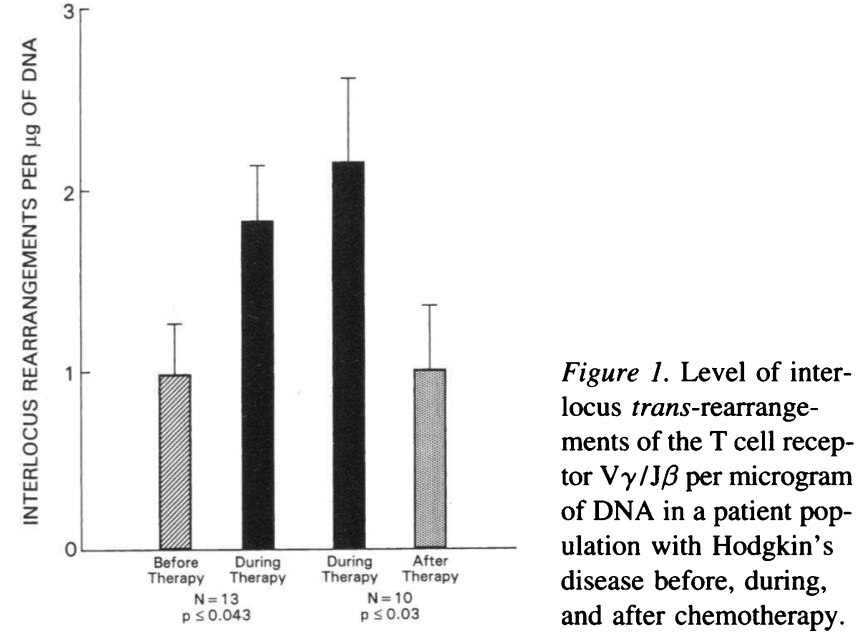

with Hodgkin's disease during their exposure to chemotherapeutic agents when compared to their prechemotherapy levels. As shown in Fig. 1, the frequency of trans-rearrangements per microgram of DNA increased during therapy from $0.98 \pm 0.28$ SE to $1.83 \pm 0.32 \mathrm{SE}$ with a $P$ value of 0.043 . 13 out of 16 patients were studied before and during therapy, 9 showed increases, 1 showed a decrease, and 3 showed no change in their level of trans-rearrangements. The frequency of trans-rearrangements per microgram of DNA decreased 1-5 mo after cessation of chemotherapy compared with values during therapy, from $2.15 \pm 0.46 \mathrm{SE}$ to $0.89 \pm 0.36 \mathrm{SE}$ with a $P$ value of 0.030 for the evaluable patients (primarily those with de novo presentation most of them treated with ABVD). Fig. 2 shows the mean level of interlocus recombination per microgram of
A

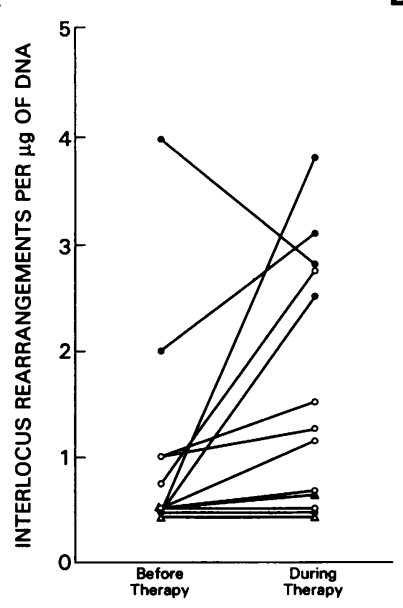

B

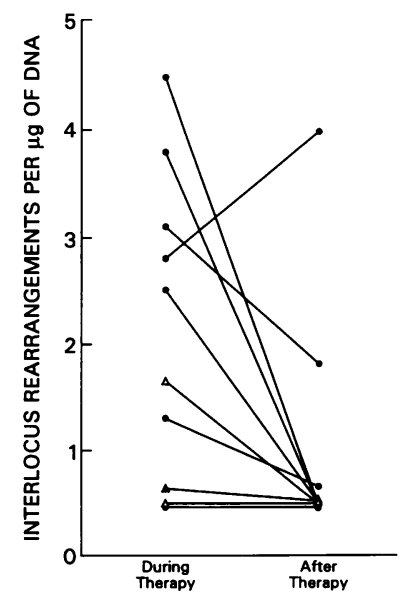

Figure 2. Level of interlocus trans-rearrangements of the T cell receptor $\mathrm{V} \gamma / \mathrm{J} \beta$ per microgram of DNA in individual patients with Hodgkin's disease in response to chemotherapy exposure, before and during therapy $(A)$, during and after therapy $(B) .1 \mu \mathrm{g}$ of DNA is equivalent to $1.5-$ $2.0 \times 10^{5}$ cells. Thus, the patient showing 4.5 trans-rearrangements per $\mu \mathrm{g}$ of DNA would be estimated as having 1 trans-rearrangement per 3.0-4.0 $\times 10^{4}$ cells. Open circles, EPOCH; closed circles, ABVD; open triangles, MOPP/ABVD and MOPP/ABV; closed triangles, MOPP. The average interval after cessation of therapy samples was 4 mo (range $0.5-9$ ). 
DNA for individual patients before and during therapy $(A)$ and during and after therapy $(B)$.

The number of patients accrued for this pilot study makes definitive comparisons based on additional subcategory stratification premature, but the data can be presented in a preliminary form. The only variables which appear to have an association with levels of trans-rearrangement are the type of therapy and patient age. Because of the circumstances of protocol eligibility, the patients treated with EPOCH had been previously treated and now had relapsed disease, whereas patients treated with ABVD were newly diagnosed. The newly diagnosed patients treated with ABVD alone had a significantly higher level of trans-rearrangements during therapy, $2.7 \pm 0.4 \mathrm{SE}$ than those treated with EPOCH $1.2 \pm 0.4 \mathrm{SE}$ with a $P=0.02$. The median age for our study group was $33 \mathrm{yr}$. When the population is stratified as younger or older than $33 \mathrm{yr}$ of age, the older patients tended to have lower levels of rearrangement during therapy, $1.2 \pm 0.4 \mathrm{SE}$ vs $2.4 \pm 0.4 \mathrm{SE}$. The difference was marginally significant, $P=0.05$. The patients with mixed cellularity type histology had higher levels of rearrangement during therapy (i.e., $P$ values between 0.1 and 0.2 ). All of the mixed cellularity patients were treated with ABVD only, and none of the relapsed patients were treated with ABVD, thus ABVD treatment may account for this difference

Although the differences do not achieve statistical significance, patients treated with EPOCH showed a more consistent but less dramatic increase in the level of trans-rearrangements during therapy than those treated with ABVD. We could not make the same comparative analysis for patients after therapy because the group that received EPOCH was treated differently, many going to autologous bone marrow transplant protocols.

\section{Discussion}

Using a PCR-based assay, we describe an increased frequency of $\mathrm{T}$ cell receptor $\mathrm{V} \gamma / \mathrm{J} \beta$ gene trans-rearrangements in PBLs of patients with Hodgkin's disease undergoing chemotherapy. The increase in trans-rearrangements was transient, returning to baseline level 1-5 mo after completion of treatment. In our study, we found that there was individual variation among patients with regard to starting level of trans-rearrangements as well as with regard to the level of trans-rearrangements in response to therapy. As shown in Fig. $2 \mathrm{~A}$, half of patients had increased levels of trans-rearrangements $>100 \%$ of their baseline level while the rest showed a $<50 \%$ increase above their baseline level. For those evaluable patients showing increased frequency of trans-rearrangements, their levels returned to baseline after therapy (Fig. 2, $A$ and $B$ ). Thus, the increased frequency of interlocus recombination between the $\mathrm{V} \gamma$ and the $\mathrm{J} \beta$ $\mathrm{T}$ cell receptor genes appears to be directly and temporally related to their exposure to chemotherapeutic agents. Our results are reminiscent of the transient acquired form of genetic instability in agricultural workers exposed to pesticides (7). Ammenheuser et al. reported (13) that, using the hypoxanthine guanine phosphoribosyl transferase assay on patients with multiple sclerosis receiving cyclophosphamide, there was a transient increase of variant frequency which returned to normal levels 7-13 wk after cessation of therapy. Chromosomal abnormalities in patients with Hodgkin's disease after therapy were previously reported by Mott et al. using the glycophorin A assay and by Caggana et al. using the hypoxanthine guanine phosphoribosyl transferase assay $(14,15)$. The latter study also suggested that there might be a group of patients with persistent chromosomal abnormalities many years after their last treatment (15).

At the population level, an increased frequency of chromosomal aberrations in the PBL may correlate with increased cancer risk for those exposed to the damaging agent (16). Only a large prospective study will allow us to enter and follow enough patients to determine whether our assay is predictive of who may develop a secondary malignancy. However, this assay does seem to be defining a general group known to be at increased risk of cancer.

We see variation among individuals with regard to "starting" level of genetic instability, response to chemotherapeutic agents, and return to baseline. At present we can not say whether the starting point, the ending point, or the "area under the curve" is most significant as a measure of genetic instability. The "gradient" hypothesis of genetic instability (17), proposes that each individual has a certain level of instability (inherited or induced), with the chromosomal breakage syndromes being at the highest end of the spectrum. The higher the level of genetic instability that an individual has, the higher his/her risk of developing cancer when exposed to a carcinogen. Individuals at the low end of the gradient are capable of dealing with large loads of carcinogen exposure, while minimal exposure will prompt individuals at the high end of the gradient to develop cancer (18).

This study suggests that measuring $\mathrm{V} \gamma / \mathrm{J} \beta$ trans-rearrangements is a rapid method to evaluate populations exposed to genome destabilizing agents. This approach has now been used to screen populations with an inherited increased risk of malignancy (4). It has also identified an increased risk of cancer because of environmental (7) and, now, iatrogenic exposure.

Increased frequency of trans-rearrangements is a measure of genetic instability and a possible marker of cancer risk. The molecular basis of this increased frequency is currently under investigation.

\section{Acknowledgments}

We are indebted to Robin Flonary and Maxine Robinson for obtaining the blood samples and to Robert Tarone for the statistical analysis of the study.

\section{References}

1. Kirsch, I. R., J. M. Abdallah, V. L. Bertness, M. Hale, S. Lipkowitz, F Lista, D. P. Lombardi. 1994. Lymphocyte-specific genetic instability and cancer. In The Molecular Genetics of Cancer. Cold Spring Harbor Symp. Quant. Biol. 59:287-295.

2. Pienta, K. J., and W. S. Ward. 1994. An unstable nuclear matrix may contribute to genetic instability. Med. Hypotheses. 42:45-52.

3. Kirsch, I. R., and S. Lipkowitz. 1992. A measure of genomic instability and its relevance to lymphomagenesis. Cancer Res. 52(Suppl.):5545s-5546s.

4. Lipkowitz, S., M. -H. Stern and I. R. Kirsch. 1990. Hybrid T cell receptor genes formed by interlocus recombination in normal and ataxia-telangiectasia lymphocytes. J. Exp. Med. 172:409-418.

5. Gatti, R. A., and R. A. Good. 1971. Occurrence of malignancy in immunodeficiency diseases. Cancer (Phil.). 28:89.

6. Boder, E. 1985. Ataxia-telangiectasia: an overview. In Ataxia-Telangiectasia: Genetics, Neuropathology, and Immunology of a Degenerative Disease of Childhood. R. A. Gatti and M. Swift, editors. Alan R. Liss Inc., New York. 163.

7. Lipkowitz, S., V. F. Garry, and I. R. Kirsch. 1992. Interlocus V-J recombina- 
tion measures genomic instability in agriculture workers at risk for lymphoid malignancies. Proc. Natl. Acad. Sci. USA. 89:5301.

8. Weisenburger, D. D. 1994. Epidemiology of non-Hodgkin's lymphoma: Recent findings regarding an emerging epidemic. Annals Oncol. 5(Suppl.1):1924.

9. Tucker, M. A., C. N. Coleman, R. S. Cox, A. Varghese, and S. A. Rosenberg. 1988. Risk of second cancers after treatment for Hodgkin's disease. N. Engl. J. Med. 318:76.

10. van Leeuwen, F. E., W. J. Klokman, A. Hagenbeek, R. Noyon, A. W. van den Belt-Dusebout, E. H. M. van Kerkhoff, P. van Heerde and R. Somers. 1994. Second cancer risk following Hodgkin's disease: a 20-year follow-up study. J. Clin. Onc. 12:312.

11. Buffone, G. J., and G. J. Darligton. 1985. Isolation of DNA from biological specimens without extraction with phenol. Clin. Chem. 31:164-165.

12. Armitage, P. 1971. Statistical Methods in Medical Research. John Wiley \& Sons Inc., New York. 116-118.

13. Ammenheuser, M. M., J. B. Ward, Jr., E. B. Whorton, Jr., J. M. Killian, and M. S. Legator. 1988. Elevated frequencies of 6-thioguanine-resistant lymphocytes in multiple sclerosis patients treated with cyclophosphamide: a prospective study. Mutat. Res. 204:509-520.

14. Mott, M. G., J. Boyse, M. Hewitt, and M. Radford. 1994. Do mutations at the glycophorin A locus in patients treated for childhood Hodgkin's disease predict secondary leukemia? Lancet. 343:828.

15. Caggana, M., H. L. Liber, P. M. Mauch, C. N. Coleman, and K. T. Kelsey. 1991. In vivo somatic mutation in the lymphocytes of Hodgkin's disease patients. Environ. Mol. Mutagen. 18:6-13.

16. Hagmar, L., A. BrØgger, I. L. Hansteen, S. Heim, B. Högstedt, L. Knudsen, B. Lambert, K. Linnainmaa, F. Mitelman, I. Nordeson, et al. 1994. Cancer risk in humans predicted by increased levels of chromosomal aberrations in lymphocytes: nordic study group on the health risk of chromosome damage. Cancer Res. 54:2919-2922.

17. Hsu, T. C. 1983. Genetic instability in the human population: a working hypothesis. Hereditas. 98:1-9.

18. Heim, S., B. Johansson, and F. Mertens. 1989. Constitutional chromosome instability and cancer risk. Mutat. Res. 221:39-51. 